ArtefaCToS. Revista de estudios de la ciencia y la tecnología

eISSN: $1989-3612$

Vol. 10, No. 2 (2021), 2. ${ }^{\text {a }}$ Época, 175-201

DOI: https://doi.org/10.14201/art2021102175201

\title{
¿Es necesaria la verdad? Una noción pragmática y deflacionaria de comprensión
}

\section{Is Truth Necessary? A Pragmatic and Deflationary Notion of Understanding}

\section{Mariano MARTÍN-VILLUENDAS}

Universidad de Salamanca, España

marianomv@usal.es

Recibido: 17/04/2021. Revisado: 25/06/2021. Aceptado: 26/10/2021

\section{Resumen}

La noción de comprensión ha sido sometida a un intenso análisis dentro de los estudios dedicados a la filosofía de la ciencia. En los últimos ańos, han surgido dos posturas claramente diferenciadas. Una primera postura, denominada veritismo, ha empleado los conceptos tradicionales de verdad y conocimiento para analizar las características básicas de la misma. La segunda postura ha considerado preciso desligar la verdad de la comprensión reseñando los componentes pragmáticos y contextuales de la misma. El objetivo del artículo será argumentar a favor de esta última postura. Para ello, se evaluará una de las principales alternativas pragmáticas que se han propuesto en los últimos años, la teoría de Henk de Regt. Se mostrará que esta postura afronta tres dificultades fundamentales que es preciso abordar a fin de justificar la postura pragmática. Con tal objetivo, se articulará una noción alternativa e innovadora de comprensión.

Palabras clave: explicación; modelos; simulación; idealización; ciencia climática.

\begin{abstract}
The notion of understanding has been subjected to intense analysis within studies devoted to the philosophy of science. In recent years, two major accounts have emerged. The first, called veritism, has employed the traditional epistemological concepts of truth and knowledge to analyze the basic characteristics of understanding. By contrast, the second account has dissociated truth from
\end{abstract}


understanding highlighting its pragmatic and contextual components. The aim of the paper will be to argue in favor of the latter account. For this purpose, one of the main pragmatic alternatives that have been proposed in recent years, the theory of Henk de Regt, will be evaluated. It will be shown that this position faces three fundamental difficulties that need to be addressed in order to justify the pragmatic position. In order to address them, an alternative and innovative notion of understanding will be articulated.

Keywords: explanation; models; simulation; idealization; climate science.

\section{Introducción}

En los últimos años, la noción de comprensión se ha convertido en uno de los principales focos de discusión dentro de los estudios dedicados a la epistemología y filosofía de la ciencia. Dentro de este último ámbito, han surgido innumerables propuestas acerca de cómo debería ser entendida, cuáles deberían ser sus condiciones de surgimiento o cuál debería ser su estatus dentro de la empresa científica. A pesar de haber sido un concepto ampliamente debatido, no existe en la actualidad un acuerdo unánime en torno a estas últimas cuestiones. Tanto es así que la discusión se ha polarizado en dos posiciones marcadamente diferenciadas: una de carácter veritista y otra de carácter pragmático/contextualista.

Del lado del veritismo se encontrarían aquellos autores que han abordado el problema de la comprensión valiéndose de las herramientas conceptuales propias de la epistemología tradicional, esto es, empleando los conceptos clásicos de verdad y conocimiento (Baumberger et al., 2019; Frigg y Nguyen, 2019; Greco, 2014; Grimm, 2006; 2008; 2012; Khalifa, 2017; Kelp, 2014; 2015; Kuorikoski y Ylikoski, 2015; Kvanvig, 2009; Rice, 2015; 2017; Trout, 2007; Strevens, 2008; 2013; Trout, 2007; Weisberg, 2013). La comprensión, sostienen estos autores, constituye una forma de conocimiento, motivo por el cual esta requiere de una justificación articulada en términos de verdad. Solo es posible afirmar que alguien comprende "p" si "p es verdadera", pues si fuese falsa difícilmente sería justificable atribuir comprensión a dicho agente cognitivo. Esta es una de las principales razones por las cuales a los defensores del veritismo se les ha denominado como "factivistas": la comprensión requiere de la posesión de conocimientos verdaderos, los cuales vienen estructurados a través de explicaciones. Este énfasis en la explicación —explicacionismo - constituye otra de las características definitorias del veritismo. Una persona dispone de comprensión solo cuando posee o capta una explicación cientifica objetiva —independiente de la psicología del agentey verdadera — capta nexos nómicos del mundo-, explicación que, en el caso de la empresa científica, suele venir articulada a través de una representación correspondentista o literalista. A este respecto, es preciso recalcar que la noción de explicación ha sido entendida, generalmente, en términos de factores/patrones 
causales o mecanismos. Una explicación verdadera debe, por tanto, reflejar los factores causales (Strevens, 2008; Weisberg, 2013; Woodward, 2003) o mecanismos (Craver, 2007) que dan cuenta del fenómeno de interés.

En la actualidad, empero, son pocos los autores que defienden una postura tan restrictiva ${ }^{1}$. Collin Rice (2015; 2021) y Christoph Kelp (2015; 2017; 2021), factivistas motu proprio, han optado por renunciar a la condición de objetividad. Cualquier análisis adecuado de la comprensión, afirman, debería tener en cuenta las consideraciones pragmáticas de los agentes cognitivos. Por su parte, los conocidos como "cuasifactivistas" han sostenido que no es necesario que las explicaciones reflejen de manera precisa todos los factores causales que intervienen en el surgimiento o historia causal del fenómeno de interés (Elgin y Sober, 2002; Kvanvig, 2009; Strevens, 2008; Weisberg, 2013; Woodward, 2003). Las explicaciones únicamente deben reflejar aquellos factores causales, mecanismos o marcadores de diferencia (difference-maker) centrales, pudiendo idealizar o eliminar aquellos factores considerados como periféricos o irrelevantes ${ }^{2}$. Las idealizaciones, desde esta perspectiva, jugarían un papel esencialmente negativo: ayudan a destacar los factores causalmente centrales (Rice, 2021).

A pesar de estos matices, es posible, sin embargo, identificar una condición mínima que engloba todos los análisis de tendencia veritista. A esta condición mínima se la suele denominar "condición de vericidad" (De Regt y Gijsbers, 2016): solo los dispositivos representacionales verdaderos, aproximadamente verdaderos o conducentes a la verdad se pueden considerar como dispositivos epistémicos legítimos y, por tanto, únicamente estos dispositivos pueden otorgar comprensión. Es decir, la verdad constituye la única condición de aceptabilidad epistémica. Si la comprensión es valiosa lo es únicamente porque está asociada con la verdad.

Esta postura veritista o (cuasi) factivista se ha visto asediada, empero, por una serie de problemas que han puesto en cuestión la validez y el alcance de su propuesta. De entre todos ellos, quizás el que más importancia tenga sea el amplio y difundido uso de idealizaciones y abstracciones dentro de la modelización científica (Jones y Cartwright, 2005; Suárez, 2009). John Greco $(2014,293)$ y Jonathan Kvanvig (2009, 342-343) han intentado abordar este problema apelando a la siguiente distinción: 1. Comprensión del modelo en sí. 2. Comprensión del fenómeno a través del modelo. Si bien la comprensión del modelo puede ser no fáctica, dado que es posible comprender modelos o proposiciones radicalmente falsas, no se puede afirmar lo mismo de la comprensión del fenómeno a través del modelo. Recordemos, no es posible comprender que "p es el caso" si p es falso.

\footnotetext{
${ }^{1}$ Como casos excepcionales tómese los análisis de J. D. Trout (2007) y Stephen Grimm (2008; 2012).

${ }^{2}$ Tome como ejemplo el modelo minimalista reseñado por Weisberg $(2013$, 100). Roman Frigg y James Nguyen (2019) constituyen una excepción. Estos autores, a pesar de sostener la verdad como criterio de aceptabilidad epistémica, rechazan el literalismo, basándose en su relato DEKI.
} 
La cuestión central es que esta división presupone que es posible tener un acceso epistémico directo a la realidad, algo que es sumamente cuestionable, dado que los fenómenos son accesibles únicamente a través de la posesión de los correspondientes dispositivos representacionales, conceptualizaciones teóricas o habilidades performativas. Estas consideraciones muestran, además, la inadecuación descriptiva de la condición de objetividad. Ya no es que el éxito de un modelo dependa de las habilidades performativas o teóricas de los investigadores, es que son precisamente las necesidades cognitivas y sociales de la investigación las que determinan qué elementos deben incluirse en el modelo y cómo debe proceder el acto de idealización (Longino, 2002).

La apelación de los cuasifactivistas a una distinción entre elementos centrales —aquellos que hacen el verdadero trabajo epistémico-y periféricos — aquellos que pueden ser idealizados o eliminados sin afectar a las virtudes epistémicas del modelo- ha sido, además, duramente cuestionada. Collin Rice $(2017 ; 2021)$ ha señalado que, en muchas ocasiones, los modelos son el foco de distorsiones holísticas, esto es, de distorsiones que tergiversan ya no solo los elementos centrales - modelo Hardy-Weinberg-, sino las mismas interacciones que se establecen entre los diversos elementos que componen el sistema (los idealizados y los no idealizados) (Rice, 2021) — modelos de optimización en biología. Son precisamente estas distorsiones holísticas que se introducen en los modelos las que permiten a los científicos emplear técnicas de modelado matemático con las que poder acceder a explicaciones que, de otro modo, serían inaccesibles. Las idealizaciones realizarían, por tanto, una contribución epistémica positiva.

De igual forma, esta postura, al articular su propuesta principalmente en torno a explicaciones causales/mecanicistas, ha pasado por alto una heterogeneidad cualitativamente distinta de explicaciones epistémicamente significativas: explicaciones matemáticas —el caso del puente de Königsberg—, explicaciones de optimización —el juego halcón-paloma - o incluso explicaciones estadísticas - los modelos de la genética de poblaciones (Lange, 2017; Reutlinger y Saatsi, 2018; Rice, 2021). El problema va más allá cuando somos conscientes de que el veritismo, al privilegiar la conexión existente entre la comprensión y la explicación - hasta tal punto de hacer depender la primera de la segunda (explicacionismo) - , ha pasado por alto una pluralidad de modelos cognitivamente relevantes sin los cuales resulta imposible comprender con profundidad el proceder científico, modelos cuyo funcionamiento epistémico prescinde del uso de explicaciones. En la sección 2.1. se mostrará, a través de un caso de estudio, hasta qué punto es preciso disociar la comprensión de la explicación (Lipton, 2013; Rice, 2016; 2021), entendiendo la segunda como una de las múltiples y posibles estrategias epistémicas que pueden impulsar la comprensión. Con ello, se pretende motivar una axiología científica que tenga como principio rector la tolerancia epistémica.

A estas consideraciones se le suma la existencia de una enorme cantidad de modelos perspectivistas que pueblan la actual práctica científica (Giere, 2006; 
Massimi y McCoy, 2020). Los científicos, con el explícito objetivo de estudiar un sistema objetivo en particular, hacen uso de una multiplicidad de modelos que, en ocasiones, sostienen asunciones incompatibles e imposibles de reconciliar ${ }^{3}$.

Todas estas dificultades han llevado a determinados autores a sostener que es preciso desligar los análisis concernientes a la verdad de los referentes a la comprensión (de Regt, 2005; 2009; 2016; 2017; Elgin, 2017; Leonelli, 2013; Potochnik, 2017; Rouse, 2015; Van Bowel, 2013). Esta última, sostienen, constituye un logro cognitivo de pleno derecho que merece ser analizado con independencia de cualquier consideración relativa a la primera. Con el objetivo de justificar la independencia conceptual de la comprensión, estos autores han propuesto analizar dicha noción tomando como referencia los intereses pragmáticos de los agentes cognitivos, sus capacidades manipulativas y las particularidades del contexto de investigación considerado. Algunos de ellos han ido más allá y han sostenido que es la comprensión, y no la verdad, el objetivo fundamental de la empresa científica (Potochnik, 2017, 91).

Llegados a este punto, la cuestión central que es preciso abordar es la siguiente: ¿por qué es necesario generar una descripción alternativa de la comprensión científica que renuncie a la verdad como aceptabilidad epistémica, haciendo énfasis en el carácter pragmático y contextualista de la misma? Aquí se establecerán dos tipos de necesidades, una de carácter descriptivo y otra normativo.

A nivel descriptivo, es preciso articular una propuesta pragmática por tres motivos. En primer lugar, para poder reflejar de manera más adecuada cómo se practica la ciencia. Como hemos visto, el veritismo tiene serios problemas a la hora de acomodar el variado uso de idealizaciones y modelos perspectivistas. En segundo lugar, para poder vislumbrar hasta qué punto las consideraciones pragmáticas influyen en las epistémicas. Algunos autores (Fraassen, 1980) han intentado mantener separadas ambas esferas. Empero, un estudio detallado de la práctica científica revela hasta qué punto los intereses pragmáticos condicionan la posibilidad misma de conocimiento y comprensión (Longino, 2002; Massimi y McCoy, 2020; Parker, 2020a; Potochnik, 2017; Rice, 2021; Winsberg, 2018). En tercer lugar, debido a que es preciso dar cuenta de la enorme heterogeneidad de intereses que encontramos en la empresa científica. Como se ha podido apreciar, los enfoques veritistas de la comprensión consideran que existe una relación muy estrecha entre esta última y la explicación. En el artículo se defenderá la existencia de una pluralidad de intereses cognitivos y objetivos heurísticos independientes de la explicación que gozan de igual estatus epistémico — tolerancia epistémica.

En cuanto a la necesidad normativa, es preciso establecer una noción de comprensión que permita generar modelos plurales, modelos que permitan avanzar

\footnotetext{
${ }^{3}$ Tome como ejemplo los modelos del cáncer (Bertolaso, 2016), del núcleo atómico (Morrison, 2011) o los climáticos (Parker, 2006).
} 
en el desarrollo científico sin imponer restricciones basadas en meras consideraciones conceptuales u ontológicas. Rasmus Winther (2020) ha afirmado que la empresa científica corre el riesgo de reificar perniciosamente las ontologizaciones que los científicos llevan a cabo con el único fin de estudiar los fenómenos ${ }^{4}$. Esto es especialmente cierto en el caso de seguir sosteniendo una actitud veritista en torno a la comprensión científica.

El objetivo del presente artículo no será otro más que argumentar a favor de una noción pragmática y contextualista de la comprensión científica. Para ello, se estructurará el mismo en tres secciones. En la primera sección, se analizará el giro pragmático llevado a cabo en torno a la comprensión. Se prestará especial atención a una de las principales propuestas pragmáticas de la actualidad, la desarrollada por Henk de Regt. De estos análisis se concluirá que la postura de de Regt afronta tres problemas esenciales que es preciso solventar si se pretende articular una noción de comprensión pragmática viable. En la segunda sección, y con el objetivo de contribuir a esta última tarea, se ofrecerá una noción de comprensión que permita superar los problemas que aquejan a la postura de de Regt.

\section{El giro pragmático de la comprensión: Henk de Regt y el criterio de inte- ligibilidad}

Debido a las dificultades conceptuales y descriptivas que afronta la posición veritista, en los últimos años diversos autores han intentado establecer formulaciones alternativas que resalten el carácter pragmático y contextual de la comprensión científica. De especial relevancia han sido las aportaciones realizadas por Henk de Regt (2005; 2009; 2016; 2017) y Catherine Elgin (2017), quienes han intentado articular de manera sistemática una postura contextualista y pragmática que tome a la comprensión como fenómeno de estudio de pleno derecho, dejando a un lado las consideraciones relativas a la verdad.

La presente sección girará en torno a la teoría delineada por de Regt. El motivo es doble. En primer lugar, por una sencilla cuestión de espacio. Analizar los pormenores de estas dos posturas requeriría de un espacio del que aquí no se dispone. En segundo lugar, porque, a pesar de haber sido elaborada de manera sistemática y de contener elementos sumamente valiosos, no ha sido discutida con tanto detalle dentro de la literatura especializada como la propuesta de Elgin 5 .

De Regt comienza su análisis afirmando que existen tres usos principales del término comprensión. 1. Sentimiento de comprensión $(F U)$ : se asocia a la

\footnotetext{
${ }^{4}$ Entiéndase por reificación confundir la representación que hacemos de los fenómenos con el mundo.

${ }^{5}$ Con ello no se afirma que la postura de Elgin no sea pertinente y que no haya influenciado la presente propuesta. Véase el monográfico dedicado en Synthese a la postura de Catherine Elgin: Themes for Elgin.
} 
experiencia fenomenológica que acompaña a la explicación 2. Comprensión de la teoría $(U T)$ : se asocia a la capacidad de emplear una teoría. 3. Comprensión de un fenómeno $(U P)$ : consiste en tener una explicación adecuada del fenómeno. A juicio de de Regt, UP constituye el objetivo epistémico de la ciencia. La cuestión central es que UP solo puede ser logrado por medio de UT. Es decir, solo se puede comprender un fenómeno en tanto en cuanto se disponga de una teoría inteligible con la cual construir los correspondientes modelos científicos. Como ya se ha mencionado, $U T$ se asocia a la capacidad de emplear una teoría — que sea inteligible para el usuario. La inteligibilidad de las teorías permite a los usuarios construir modelos con los cuales poder derivar explicaciones de los fenómenos en cuestión. Para construir estos modelos se requiere de habilidades — para relacionar las teorías y modelos_y de juicios — para establecer qué idealizaciones y abstracciones son pertinentes. Para de Regt no es suficiente con que un agente disponga del conocimiento, es preciso que este sepa usarlo, aplicarlo. Qué habilidades o juicios son adecuados dependerá de la teoría y de las necesidades cognitivas de los agentes. Con estas consideraciones, de Regt pone en el centro de su teoría a los agentes recogiendo los reclamos fundamentales establecidos por los teóricos de la virtud (Pritchard, 2009; Sosa, 2007): la comprensión implica cierto logro cognitivo.

Dado que la comprensión de las teorías es fundamentalmente pragmática, pues requiere de habilidades y de juicios, de Regt concluye que la condición de objetividad no puede ser aplicada a UP. UP es pragmática y contextualista porque $U T$ también lo es. Tomando como base estas consideraciones, de Regt establece su criterio fundamental para la comprensión de un fenómeno $(C U P)$ :

Un fenómeno $\mathrm{P}$ es comprendido científicamente si y solo si existe una explicación de $\mathrm{P}$ que esté basada en una teoría inteligible $\mathrm{T}$ y se ajuste a los valores epistémicos básicos de adecuación empírica y consistencia interna $(2017,93)$.

CUP, que constituye la base de la teoría de la comprensión propuesta por de Regt, tiene como condición esencial la inteligibilidad. Esta condición hace referencia al valor que los científicos atribuyen al conjunto de cualidades o virtudes que facilitan el empleo de una teoría T para la construcción de modelos (2017, 40). La inteligibilidad no es una propiedad intrínseca de las teorías, sino que es una propiedad contextual, es decir, depende no solo de las cualidades inherentes de la teoría sino de las habilidades de los científicos en cuestión, habilidades que se adquieren durante el proceso formativo. Diferentes contextos de investigación pueden requerir el ejercicio de diferentes tipos de habilidades. Es por este motivo por el que de Regt afirma que el valor epistémico de las teorías no puede ser evaluado en aislamiento, sino que debe ser evaluado en relación con los agentes. Determinada clase de agentes puede preferir emplear una teoría frente a otra debido a que disponen de las habilidades y conocimientos necesarios para emplearlas, o 
sencillamente porque consideran que sus virtudes son superiores. Comprender un fenómeno P sobre la base de una teoría T depende, por tanto, de una combinación adecuada de las habilidades de los sujetos y de las cualidades de las teorías.

A partir de estas consideraciones, de Regt sigue a Margaret Morrison (2015) en sus reflexiones sobre la imposibilidad de establecer una definición precisa de comprensión. Por ello, establece que su noción posee un marcado carácter deflacionario - capta únicamente condiciones necesarias: CUP establece, únicamente, que los agentes deben emplear teorías que sean inteligibles, siendo el contexto el elemento que determina dicha inteligibilidad. Resulta, por tanto, imposible delinear una definición de comprensión algorítmica que especifique de manera detallada y para todos los contextos cuándo una teoría se considera inteligible.

\subsection{Problemas y tensiones en la propuesta de de Regt}

Si bien es cierto que la propuesta de de Regt es capaz de dejar a un lado los problemas propios del veritismo al adoptar la condición de inteligibilidad, no es menos cierto que esta se ve asediada por tres problemas no menos importantes que es preciso abordar. El primer problema es de carácter descriptivo. Los restantes, de carácter filosófico.

Vayamos con el problema descriptivo. A juicio de de Regt, la comprensión científica de un fenómeno precisa de la capacidad de usar una teoría de tal forma que permita al usuario construir un modelo del cual se pueda derivar una explicación. La cuestión central, y el primer gran problema de la teoría de de Regt, es que esta es incapaz de dar cuenta de una clase especial de modelos que están gozando en la actualidad de una amplia consideración dentro de la comunidad científica: modelos que se caracterizan por desplegar una opacidad epistémica y una independencia parcial de la teoría. Como ejemplo de estos últimos tenemos los modelos climáticos (Frigg et al., 2015a; 2015b; Lenhard, 2019; Lenhard y Winsberg, 2010; Parker, 2006; 2014; 2020a; 2020b; Winsberg, 2010; 2018).

El modelado climático constituye una ciencia extraordinariamente compleja que tiene por objetivo elaborar una serie de predicciones cuantitativas acerca del cambio climático. Se afirma que el sistema climático es un sistema complejo debido a que los procesos causales que vertebran el mismo (dinámica de las capas de hielo, circulación atmosférica, formación de nubes, corrientes oceánicas, entre otros) son numerosos, interactivos y no lineales — no se pueden abordar de manera directa usando las herramientas matemáticas clásicas. Es por este motivo por el que los científicos se ven obligados a hacer uso de modelos de simulación. Estas herramientas computacionales permiten explorar el comportamiento aproximado del modelo matemático estimando soluciones a las ecuaciones originales — sobre la base de unas condiciones iniciales y de contorno dadas. 
Los modelos climáticos se suelen construir a partir de una serie de ecuaciones diferenciales parciales. Tal y como se ha mencionado, estas ecuaciones son intratables usando métodos analíticos, por lo que se discretizan en una cuadrícula (grid). Es decir, se convierten en ecuaciones de diferencia discretas que aproximan las primeras. Establecidas estas consideraciones preliminares, conviene tener en cuenta dos puntos estrechamente relacionados. El primero, que al realizar este procedimiento de discretización no se parte de una representación fiel y precisa del mundo. La discretización solo permite describir algo que se aproxime a la solución de las ecuaciones continuas originales, en cierto grado de precisión - marcado por las exigencias pragmáticas del proyecto de investigación (Winsberg, 2018, 40). Tal y como indica Eric Winsberg, siempre existirá cierta incertidumbre en relación con el proceso de discretización. La transición de la descripción continua, provista por las ecuaciones originales, a la representación discreta y finita, dada por las ecuaciones de diferencia, origina lo que se conoce como el problema del truncamiento. Este problema hace referencia a la cuestión de encontrar un procedimiento de aproximación numérico adecuado que permita dar con una dinámica estable. Estas consideraciones ponen en cuestión el aspecto veritista de la comprensión: el modelado climático incluye elementos artificiales/pragmáticos que son indispensables para lograr la actuación deseada del sistema. El segundo punto hace referencia a la parametrización. Cuando se discretizan, los modelos de simulación poseen un tamaño de cuadrícula finito. Todas aquellas interacciones que tengan lugar por debajo de la escala de la cuadrícula (grid) no pueden calcularse por medio de las ecuaciones discretizadas. Por ello, es preciso usar un modelo subcuadrícula (sub-grid). A esto se le conoce, dentro de la literatura dedicada al modelado climático, como parametrización (tome la formación de nubes como un caso paradigmático de parametrización) (Winsberg, 2019). Es preciso reseñar, además, que con estos procedimientos matemáticos los modeladores no pretenden construir una representación que refleje de manera precisa — determinados aspectos de - la realidad. De hecho, son estas distorsiones generalizadas, introducidas a través de dichos dispositivos matemáticos, las que permiten a los científicos ajustar sus representaciones a las herramientas de las que disponen dentro de su correspondiente esfera epistémica, pudiendo, de esta manera, extraer información potencialmente significativa del fenómeno (Rice, 2021).

¿En qué medida el modelado climático pone en cuestión las consideraciones establecidas por de Regt? En línea con las reflexiones establecidas por Johannes Lenhard $(2013$; 2019), se sostendrá que es posible lograr comprensión a partir de modelos epistémicamente opacos, esto es, a partir de modelos no inteligibles. Antes de llegar a esta conclusión es preciso examinar qué es la opacidad epistémica y por qué se dice que los modelos climáticos tienen este rasgo como característica distintiva (Durán y Formanek, 2018; Humphreys, 2004; Lenhard, 2019). Se dice que un proceso es epistémicamente opaco para un determinado agente 
epistémico cuando este es incapaz de examinar - tener acceso a - los elementos epistémicamente relevantes del mismo —a los pasos de justificación, en palabras de Juan Durán y Nico Formanek (2018)—.

La primera causa de la opacidad epistémica es algo que ya se ha mencionado: la complejidad de los modelos climáticos. Estos modelos computacionales cuentan con innumerables pasos justificativos imposibles de supervisar por parte de los agentes humanos. Se han seńalado dos causas principales por las que es imposible verificar de manera pormenorizada los procesos computacionales (Durán y Formanek, 2018). 1. La velocidad: ningún agente humano es capaz de seguir el ritmo de los procesos computacionales. 2. Volumen de datos: la memoria de los agentes cognitivos es finita y limitada.

La segunda causa es lo que Lenhard y Winsberg (2010) han denominado como "modularidad difusa". Herbert Simon, en su famoso libro, The Sciences of the Artificial, estableció que la modularidad constituía un concepto central dentro de los estudios dedicados a los sistemas complejos. La razón de ello de debe a que este concepto permite abordar, de manera racional, el tratamiento de tales sistemas. Grosso modo, la modularidad establece el grado en el cual las tareas complejas pueden ser divididas en tareas más simples, o de manera más general, el grado en el cual los componentes de un sistema pueden ser fraccionados en subcomponentes. Por tanto, el concepto de modularidad apunta a establecer el grado de confianza a través del cual es posible evaluar el comportamiento de cada subcomponente o subtarea por separado. Empero, la modularidad hace referencia a un segundo proceso: a la capacidad de reconstruir o recombinar de manera aditiva esos componentes o tareas a fin de comprender el funcionamiento del sistema en su conjunto (Simon, 1996, 128). Una creencia firmemente establecida en relación con los modelos climáticos es que estos se construyen sobre la base de tal modularidad: constan de diferentes módulos independientes que se conectan a través de un acoplador. Lenhard y Winsberg (2010) han cuestionado esta última idea argumentando que los modelos climáticos se caracterizan por una "modularidad difusa". Este término hace referencia a la imposibilidad de evaluar por separado las virtudes y fallas de cada uno de los módulos que integran los sistemas climáticos - intratabilidad analitica. Existen dos razones por las que se alza esta característica. La primera, debido a la complejidad de las interacciones que se establecen entre los diferentes módulos. A juicio de estos autores, no es que primero se obtengan los resultados en cada uno de los módulos (circulación atmosférica, dinámica de hielo o formación de nubes) y luego se sinteticen a través de un acoplador, sino que cada módulo intercambia sus resultados con los restantes módulos durante el tiempo que dura la simulación. En segundo lugar, debido al proceso de calibración. Estos autores afirman que la determinación de qué parametrizaciones son las más adecuadas depende del comportamiento general del modelo, esto es, de cómo se han representado los restantes procesos subcuadrícula y cuáles han sido los errores que estos han introducido en la dinámica general. Existe, por tanto, lo que estos autores denominan como 
"dependencia de ruta": "el siguiente paso del modelado está influenciado por los efectos acumulados de los pasos previamente implementados" (Winsberg y Lenhard, 2010,256). Resulta imposible saber si los ajustes que se han establecido sobre los valores de los esquemas de parámetros son buenos porque ajustan el comportamiento general del modelo (compensando otros fallos) o porque corrigen errores discretos. Esta "modularidad difusa" puede ser vista como una consecuencia de lo que Rice (2021) ha denominado como "distorsiones holísticas", distorsiones que afectan, no ya solo a los elementos epistémicamente centrales del modelo, sino a las mismas interacciones que se establecen entre los elementos que lo componen ${ }^{6}$.

La tercera causa está estrechamente relacionada a la segunda — calibracióny es la plasticidad característica de estos modelos. Como ya se ha afirmado, la calibración constituye un elemento crucial dentro del modelado climático porque permite adaptar la dinámica de la simulación con el objetivo de mejorar el rendimiento del modelo. La cuestión es que esta calibración incrementa la opacidad del sistema al ocasionar que propiedades de la dinámica final del mismo no se deriven de la estructura teórica de modelo, sino de los mismos ajustes y adaptaciones que los modeladores han llevado a cabo durante el tiempo que dura la simulación, adaptaciones que, como ya hemos visto, disponen de un carácter marcadamente holístico. La dinámica global del sistema no puede ser derivada, por tanto, de la estructura teórica. Aquí el rol heurístico o exploratorio juega un papel crucial. Al llevar a cabo sucesivas iteraciones, los modeladores examinan, de manera práctica, un espacio de posibilidad no fáctico — modal - que es, sin embargo, significativo en términos cognitivos y epistémicos. Con ello los investigadores abren nuevas posibilidades de trabajo, nuevos escenarios de investigación, nuevas posibilidades de intervención sobre la realidad o nuevos pasos inferenciales —incluso posibles explicaciones - desconocidos hasta el presente momento. Al explorar, de manera práctica, esa esfera modal y no fáctica del espacio de posibilidad asociado a un determinado fenómeno, los investigadores contribuyen de manera significativa a la compresión del fenómeno en cuestión. Este hecho pone en cuestión el requisito de inteligibilidad de las teorías establecido por de Regt: ya no es preciso tener una teoría inteligible para poder obtener comprensión de un fenómeno a través de un modelo. La teoría pasa a un segundo plano y el carácter exploratorio, iterativo y modal del modelado adquiere una importancia primaria.

Tomando estas consideraciones, es posible afirmar dos cosas. En primer lugar, que los usuarios epistémicos son capaces de lograr cierta comprensión del fenómeno de manera independiente a la teoría, y de manera más general, de manera independiente a la explicación (Morgan y Morrison, 1999; Rice, 2021; Winsberg, 2006). Ello se logra iterando, explorando, manipulando e interviniendo sobre el modelo de simulación. Con ello, los investigadores, aun sin tener una explicación del fenómeno —una respuesta a una pregunta por qué-, pueden

${ }^{6}$ Esta consideración reincide en el carácter no fáctico del modelado climático. 
concebir escenarios hipotéticos plausibles, escenarios incompatibles, cadenas/relaciones inferenciales novedosas que puedan resultar en nuevas preguntas, usos o afirmaciones de posibilidad e incluso posibles intervenciones sobre la realidad -basándose en el estudio de la evolución de los sistemas de simulación que cuentan con un rango determinado de parámetros. En segundo lugar, que los modelos deben considerarse como objetos epistémicos de pleno derecho que poseen cierto grado de independencia de la teoría. Como hemos visto a través del problema de la discretización, los modelos disponen de sus propios problemas epistémicos y metodológicos.

Los problemas restantes son de naturaleza filosófica y hacen referencia a la naturaleza de la relación entre la explicación y la comprensión y a la cuestión de los grados de comprensión.

De Regt asocia estrechamente la comprensión a la explicación hasta el punto de hacer depender la primera de la segunda $(2017,263)$. Incurrir en esta suerte de "explicacionismo" no hace sino mermar la profundidad conceptual y el carácter pragmático de su propuesta, pues obvia la enorme variedad de intereses y objetivos cognitivos que pueblan la práctica científica. Al establecer este requerimiento, de Regt está alineándose con una larga tradición filosófica que, de manera no casual, se ha asociado con las perspectivas veritistas (Greco, 2014; Khalifa, 2017; Kuorikoski y Ylikoski, 2015; Strevens, 2008; 2013; Trout, 2007). De Regt, no obstante, sostiene, sobre la base del criterio de inteligibilidad, un "explicacionismo moderado" de base pluralista. Es decir, de Regt proporciona una definición de comprensión que es independiente de cualquier modelo particular de explicación. A su juicio, diferentes agentes epistémicos pueden adquirir comprensión por medio de diferentes modelos o estrategias explicativas (nomológico-deductivo, unificacionista, manipulacionista, intervencionista, mecanicista o kairética, entre otros). Qué estrategias explicativas sean las más adecuadas para lograr un determinado objetivo explicativo dependerá del contexto y de las habilidades particulares de los científicos en cuestión.

Si bien es cierto que de Regt no hace depender la comprensión de un modelo particular de explicación, no es menos cierto que sí que la hace depender de la noción general de explicación. De esta manera, de Regt sacrifica la idea de un pluralismo profundo — un pluralismo que sepa reconocer la variedad de intereses cognitivos de los distintos investigadores - por la de un pluralismo explicativo. Esto hace que la propuesta de de Regt sea, en cierta medida, ineficiente a nivel conceptual. Nos enfrentamos a una disyuntiva: o consideramos que la propuesta de de Regt es limitante o negamos el estatus cognitivo y epistémico a una amplia gama de modelos científicos. Claramente, los modelos climáticos han sido valiosos a nivel epistémico dado que han permitido arrojar luz sobre las dinámicas subyacentes al cambio climático. Han permitido examinar un espacio de posibilidad sumamente relevante a nivel científico, social y económico: han explorado qué escenarios son posibles, cuáles son inconcebibles, han detallado 
posibles dinámicas climáticas (resultados) dado cierto rango de parámetros, han determinado cómo cambios particulares pueden conducir a cierta clase de resultados, han establecido, mediante constreñimientos estructurales, por qué ciertas dinámicas parecen necesarias e incluso delineado posibles medidas de intervención sobre la realidad basándose en modelos particulares de simulación. Este último aspecto es crucial: los modelos climáticos han resultado ser enormemente útiles como herramientas de intervención sobre la realidad, especialmente a la hora de diseñar políticas climáticas. Si bien la explicación — respuesta a preguntas por qué (Why) — resulta ser un objetivo cognitivo sumamente importante que impulsa nuestra comprensión de la realidad, no por ello se puede afirmar que la comprensión se reduce a la explicación (Rice, 2021). La exploración modal de espacios de posibilidad (regiones heurísticas) — respuesta a preguntas "cómo es posible" (How) — resulta ser igualmente importante. Estas respuestas pueden, al igual que la explicación, impulsar nuestra comprensión de la realidad. La cuestión de fondo es que la noción de de Regt parece ser demasiado estrecha: no tiene en cuenta la existencia de objetivos cognitivos alternativos a los de la explicación — tolerancia epistémica.

Vayamos con el segundo problema. De Regt no aborda de manera explícita el problema de los grados de comprensión. Esto se debe, fundamentalmente, a que, si bien de Regt afirma que no es preciso tener una teoría completamente articulada para poder tener comprensión, no específica nada acerca de si es posible que la inteligibilidad de las teorías, elemento sobre el cual se vertebra CUP, admita graduaciones. Dicho esto, es posible adoptar dos actitudes hacia la teoría de de Regt.

Una primera actitud consistiría en dar por sentado que su teoría concibe la comprensión como un asunto dicotómico: o se tiene comprensión o no se tiene. Esta actitud, parece ser, en cierta medida, la más natural. Resulta un tanto extraño concebir la idea de que una teoría sea más o menos inteligible, parcialmente inteligible o lo suficientemente inteligible. ¿Quién o qué determina que una teoría sea lo suficientemente inteligible para un agente? ¿Dónde se establece el límite entre lo inteligible y lo no inteligible? Parece que un usuario cuenta o no cuenta con las habilidades y juicios para construir un modelo. Que es capaz, o no, de emplear el modelo. Que es capaz, o no, de derivar explicaciones a partir del mismo. Tomando esta actitud, es posible señalar dos críticas a la teoría de de Regt. La primera es que su propuesta no consigue reafirmar el carácter contextual y pragmático de la comprensión, pudiéndose distanciar, así, del espectro veritista. La comprensión explicativa constituye el summum bonum. O se tiene comprensión, y en este caso explicativa, o no se tiene comprensión. Todos aquellos grados intermedios — casos de comprensión asociada a otros objetivos cognitivos como la predicción, casos de comprensión imperfecta o casos de comprensión sin mediación teórica - no contarían como verdadera comprensión. La segunda, que la teoría de de Regt describe, de manera bastante deficiente, la práctica científica real. Como se pudo comprobar en el caso de los modelos climáticos, la 
comprensión no es un asunto de todo o nada. Los científicos no construyen el modelo y ganan comprensión del fenómeno de manera absoluta y directa. Más bien, lo que ocurre es que se gana comprensión de manera progresiva a medida que se realizan las calibraciones y ajustes necesarios en el modelo, manipulándolo y explorando las posibilidades cognitivas que este ofrece. De Regt obvia por completo el rol heurístico y modal de la comprensión.

Una segunda actitud sería asumir que de Regt ha dejado, sencillamente, sin clarificar este último punto. En este caso, y con el objetivo de corregir las inadecuaciones de su teoría, debería ofrecer una respuesta a las siguientes cuestiones: ¿Es posible tener una teoría más o menos inteligible? ¿Dónde y de qué manera se establece el criterio mínimo de inteligibilidad? En el mejor de los casos, la teoría de de Regt está incompleta y debería especificarse si es posible concebir grados de inteligibilidad y de qué manera. En el peor de los casos, la teoría de de Regt es inadecuada a nivel descriptivo e ineficiente a nivel conceptual.

\section{3. ¿Es posible, entonces, adoptar una actitud pragmática en relación con la comprensión?}

Analizados los problemas concernientes a la noción de comprensión de de Regt, cabría preguntarse, ¿es posible ofrecer una alternativa pragmática y contextualista viable de la compresión? En esta sección se ofrecerá una respuesta afirmativa.

Es preciso, sin embargo, establecer unas consideraciones preliminares. En primer lugar, no se pretende establecer una definición de comprensión al uso, esto es, delinear cuáles son las características fundamentales de la comprensión. Lo único que se pretende es establecer las condiciones de contorno que rodean a la misma, sus aires de familia (Wittgenstein, 2015, 49). O, dicho de otra manera, el conjunto de condiciones necesarias — no suficientes - por las que es posible afirmar que un agente cognitivo tiene comprensión.

Por mera inteligibilidad conceptual, aquí se empleará el término noción en lugar de definición para hacer referencia a la presente propuesta, debido al carácter general y abierto que se le puede adscribir a dicho término. El objetivo de la sección será, por tanto, establecer una noción deflacionaria ${ }^{7}$, pragmática, contextualista y minimalista ${ }^{8}$ de comprensión: Se dice que alguien tiene comprensión cuando (1) existe la condición de posibilidad para poder (2) ejercer una(s) determinada(s) capacidad(es) (3) fundada(s) sobre los estándares de aceptación de una subcomunidad

\footnotetext{
${ }^{7}$ Por deflacionario entiendo que únicamente capta condiciones necesarias (Suárez, 2010). No es posible definir universalmente las condiciones de aplicación de la comprensión dado que esta está vinculada al uso.

${ }^{8}$ Por minimalista entiendo que no tiene una propiedad o estatus teórico sustancial (Price, 2011, 115-117) más allá de su papel dentro de las prácticas discursivas humanas.
} 
epistémica de agentes, con el objetivo explícito de satisfacer, de manera adecuada, (4) ciertos intereses/necesidades cognitivas (5) de manera eficiente y coherente (lógica, empirica, metodológica y epistémicamente)

La cláusula (1) señala el carácter eminentemente contextual de la noción propuesta: para poder adquirir comprensión y para poder desplegarla/manifestarla es preciso que se den las condiciones habilitantes pertinentes. Esta primera condición pretende acomodar una crítica establecida por Kelp (2015) al respecto de las teorías manipulacionistas de la comprensión. Kelp ilustra su crítica a través del denominado "caso de los agentes disléxicos". Estos agentes podrían ser capaces de comprender un fenómeno incluso si no lo pudiesen reflejar. Esta cláusula permite, por tanto, abordar las críticas establecidas por Kelp sin renunciar al manipulacionismo propio de las propuestas pragmáticas.

Las cláusulas (3) y (4) estipulan que la comprensión no constituye un fenómeno ocioso. Esta es dependiente de unas condiciones materiales y epistémicas muy concretas. Se da siempre en el seno de una subcomunidad' particular de agentes cognitivos. Por ello, sus condiciones de posibilidad, de ejercicio y de modalidad tienen lugar en un entorno de investigación complejo que incluye una amplia variedad de elementos epistémicos y no epistémicos que estructuran y determinan lo que se considera válido (condición de posibilidad o región asertiva), significativo (condición de ejercicio o región normativa) y posible (condición de modalidad o región modal) (Ankeny y Leonelli, 2020, 52). Antes de analizar estas últimas condiciones, veamos en qué consisten los elementos epistémicos y no epistémicos que delinean los contornos de la comprensión.

Los elementos epistémicos hacen referencia al conocimiento - ya aceptado por una subcomunidad - que un agente adquiere durante su etapa de instrucción. Sabina Leonelli (2013) ha realizado una distinción analítica entre dos clases de conocimiento: conocimiento teórico y conocimiento corporeizado/performativo. Con esta partición, Leonelli ha intentado poner de manifiesto una idea que se ha tratado de repetir a lo largo del artículo: el conocimiento teórico - inteligibilidad - no determina los resultados del modelado y, por ende, tampoco el surgimiento de la comprensión. El conocimiento teórico comprendería los conceptos, las teorías, los modelos disponibles, el estado de la cuestión, las explicaciones, los conceptos, los métodos de investigación, los objetivos o las cuestiones de interés ya aceptadas por parte de la subcomunidad. La cuestión central es que, si entendemos la comprensión como el ejercicio de una determinada capacidad, como un cierto tipo de saber-cómo (Brandom, 1994/2005; 2009; 2011), entonces esta no puede limitarse únicamente a la captación de cuerpos intralingüísticos de información, esto es, a la posesión de una constelación de creencias coherentes.

\footnotetext{
${ }^{9}$ Nótese que no se empela el término comunidad, el cual hace referencia a núcleos epistémicos más amplios (como la biología, física o química, por ejemplo). El término subcomunidad, por el contrario, hace referencia a núcleos de investigación más reducidos como la microbiología, la bioinformática, la genética poblacional o la biología de sistemas, entre otros.
} 
El conocimiento corporeizado hace referencia a las habilidades performativas que obtienen los agentes a través de su instrucción. Esto incluye el conocimiento sobre los procedimientos y protocolos de intervención, habilidades en el empleo de instrumentación, el conocimiento de los modos/medios de presentación más adecuados o la experticia en el calibrado, ajuste y manipulación de modelos. Esto no significa, por otra parte, que el conocimiento performativo se reduzca únicamente a la aplicabilidad del conocimiento teórico. El conocimiento performativo constituye una puerta de acceso epistémico de pleno derecho a los fenómenos de interés (Hacking, 1983). Tal y como se mencionó en la sección anterior al respecto de los modelos climáticos, este conocimiento performativo resulta esencial porque permite, gracias a su carácter exploratorio e iterativo, abrir un espacio heurístico de investigación que conduce a la reconfiguración de los contornos del dominio de investigación —espacio de razones (Sellars, 1956/1997)— de una determinada subcomunidad, pudiendo, de esta manera, cubrir algunas de las lagunas que deja el conocimiento teórico (Lenhard, 2013, 173). Tómese el caso de los organismos modelo (Ankeny y Leonelli, 2020). Si bien es cierto que estas prácticas se fundamentan sobre el conocimiento teórico acerca del papel que juega la conservación evolutiva — genética o mecanicista—, aquí el conocimiento teórico no agota la obtención posible de comprensión. Determinadas prácticas performativas y exploratorias resultan ser esenciales. Como ejemplo de prácticas performativas tome la estandarización de los entornos que rodean a los organismos modelo (ambientales o genómicos), la priorización de determinados rasgos o la misma alteración de los genes, mecanismos o elementos de interés. Como ejemplo de prácticas exploratorias tome los denominados casos de Knockout genético en donde se "desactiva" un gen para entrever las posibles consecuencias y, por ende, los efectos del mismo. El acceso epistémico al fenómeno de interés, la comprensión del mismo, viene determinada, en gran parte, por el espacio heurístico o de posibilidad que abre la manipulación material del organismo en cuestión. A través de esa manipulación los investigadores pueden adquirir comprensión acerca de formas de vida diferentes. Rachel Ankeny y Leonelli (2020) han señalado cómo la investigación de las vías de seńalización del pez cebra $D$. rerio resultó ser crucial a fin de adquirir comprensión sobre la aparición de la enfermedad de Alzheimer o cómo se empleó el ratón knockout p53 para estudiar la proliferación y diseminación de una amplia variedad de cánceres humanos. La manipulación de estos organismos permite explorar nuevas rutas, nuevas vías de investigación, nuevos espacios de posibilidad que de otro modo habrían sido imposibles - por la complejidad de los organismos, por consideraciones éticas o financieras. El caso de los organismos modelo, al igual que el de los modelos climáticos, reseńa la importancia del conocimiento no teórico: las habilidades performativas que proceden a través de un comportamiento manipulativo, exploratorio e iterativo resultan ser extremadamente importantes. Con esto no se pretende afirmar que la comprensión sea exclusivamente performativa. Lo que se pretende es resaltar la importancia de un elemento que, usualmente, ha sido pasado por alto. 
Por su parte, los elementos no epistémicos incluirían la estructura institucional y pedagógica — como universidades o institutos de investigación-, la estructura de comunicación — como revistas o conferencias-, de financiación — como agencias estatales o privadas —, la infraestructura material — como laboratorios o departamentos - o social — que determina la forma de distribuir el trabajo o los comportamientos adecuados. De esta manera, es posible afirmar que la comprensión se ve condicionada y moldeada por una heterogeneidad de factores no epistémicos. Es la subcomunidad, en su conjunto, la que establece tanto las condiciones de generación como de aceptación de la comprensión (Elgin, 2017, 110). Winsberg, al respecto de la discusión sobre la parametrización, ha señalado que existen opciones metodológicas epistémicamente no forzadas que se hallan determinadas por valores sociales — priorizar la simulación precisa de cambios en la temperatura de la superficie en base a su importancia social o económica.

A fin de comprender la cláusula (2) es preciso considerar las condiciones para la comprensión reseñadas unas líneas más atrás. La condición de posibilidad reseña la idea de que, para poder tener la posibilidad de adquirir comprensión, los agentes cognitivos deben instruirse, reconocer, anclarse y responder de manera dialógica a los elementos epistémicos y materiales que conforman la subcomunidad. Es decir, al entender la comprensión como una capacidad/habilidad, como un saber-cómo, se pretende recalcar dos puntos. El primero, que la comprensión se da siempre en relación con un agente inserto en una subcomunidad. Exige que un agente dirija su mirada atenta hacia un problema que considera interesante, algo que viene posibilitado por su pertenencia a una subcomunidad. A diferencia de la condición de objetividad propia de las propuestas veritistas, sostengo que carece de sentido hablar de comprensión si no se especifica el agente, sus necesidades cognitivas y las condiciones materiales y epistémicas que rodean al mismo. En segundo lugar, que los agentes deben adquirir cierto saber-cómo, una maestría en el rol inferencial dentro de la subcomunidad (Brandom, 1994/2005; 2009; 2011), lo cual no es otra cosa más que saber cómo hacer inteligible un cuerpo de creencias y prácticas ya instituido. Esto es, saber emplazar dentro de una red de relaciones inferenciales —espacio de razones (Sellars, 1956/1997) — los aspectos cognitivamente significativos ${ }^{10}$. Esto implica aprender a distinguir qué se sigue - o no- de qué, a cómo clasificar las inferencias correctas y las incorrectas, así como a conocer la evidencia existente a favor y en contra de las mismas. Esta condición constituye la región asertiva porque, desde un plano personal, está orientada a que el individuo pueda articular de manera conceptual y material (Rouse, 2015) aquellos aspectos cognitivamente significativos marcados por la subcomunidad y porque, desde un plano social, está orientada a racionalizar y reafirmar la comprensión ya asentada por parte de los predecesores, delimitando,

\footnotetext{
${ }^{10}$ Esto incluye tanto aspectos intralingüísticos (teóricos), como prácticos y materiales (performativos).
} 
así, de manera más precisa los contornos de la subcomunidad. Con estas consideraciones se incorporan, además, las condiciones de comprensión reflexiva establecida por Duncan Pritchard $(2010,82)$ — los agentes deben poseer fundamentos reflexivamente accesibles que apoyen las creencias relevantes que sustentan su comprensión-y de coherencia señalada por Elgin (2017) —la justificación epistémica requiere cierto equilibrio reflexivo.

La condición de ejercicio reseńa las posibilidades u objetivos de investigación abiertos y significativos establecidos por una determinada subcomunidad sobre los que un agente cognitivo trabaja para poder articular la inteligibilidad del correspondiente dominio de investigación. Corresponde a la región normativa porque se halla orientada a lograr la inteligibilidad de una serie de aspectos significativos, lo cual exige de una interacción dialógica: los agentes deben dar las razones que apoyen o justifiquen sus afirmaciones y estas, a su vez, deben poder ser tomadas por otros miembros como premisas para sus propias inferencias (Brandom, 1994/2005; 2009; 2011; Rouse, 2015). Esta es la principal razón por la cual la comprensión es fundamentalmente activa - tanto en su sentido de posibilidad como de ejercicio. Un agente ejerce una capacidad con el explícito objetivo de satisfacer, de manera efectiva, ciertos objetivos cognitivos. Para ello, debe ser capaz — saber-cómo- de ofrecer razones que la justifiquen frente a los demás miembros. Qué aspectos sean significativos, cuáles sean esas razones particulares y cómo se materialice el saber-cómo es algo que depende de cada contexto de investigación y subcomunidad particular. Este hecho es, esencialmente, el que marca la frontera que separa las distintas subcomunidades epistémicas que articulan la empresa científica.

La condición de modalidad reseña el carácter inacabado, dinámico y perfectible de la comprensión. Como hemos visto, la comprensión se estructura a través del ejercicio de cierta(s) capacidad(es), contextualmente dependiente(s), a través de un saber-cómo — maestría en el rol inferencial—, y no a través de la posesión o captación de un conocimiento o explicación verdadera, de un saber-qué. La comprensión no es, pues, un estado, no se articula a través de la posesión de nada. Es una habilidad, una capacidad, una cierta maestría en el rol inferencial dentro del espacio de razones articulado por los elementos epistémicos y no epistémicos de una subcomundidad. Esto incluye, además de las capacidades ya mencionadas en la condición de posibilidad y de ejercicio, el hecho de ser capaz de explorar qué es posible y qué no lo es. Al hacer explícitos los compromisos en el seno de una subcomunidad, al explorar las consecuencias inferenciales intralingüísticas y materiales, en definitiva, al actuar, los individuos modifican, extienden y reconfiguran el dominio de investigación, su espacio de razones, abriendo, de esta manera, nuevas posibilidades de investigación y comprensión (Brandom, 1994/2005; 2009; 2011; Longino, 2002; Rouse, 2015). Esto les permite generar un espacio heurístico de trabajo que permite hacer significativos nuevos aspectos de la realidad, pudiendo, en último término, trabajar para lograr una mayor inteligibilidad del dominio de investigación. Con esta idea se satisface 
un requerimiento establecido por Kelp (2015): es necesario reconocer que, en un tiempo futuro T2, un agente pueda tener una comprensión mejorada/más completa/más inteligible del fenómeno de interés

Con estas consideraciones se resaltan tres aspectos esenciales de la noción de comprensión propuesta. En primer lugar, que la comprensión es fundamentalmente comprensión social y no individual. En esto me opongo al marcado carácter individualista que ha caracterizado a las propuestas veritistas de la comprensión. $\mathrm{Al}$ tener sus condiciones de posibilidad, de ejercicio y de modalidad en la subcomunidad, al ser un saber-cómo basado en hacer explícitos los compromisos y legitimaciones, al tener que someterse al juego de dar y pedir razones no es posible hablar de una comprensión tácita, algo que algunos autores han tratado de defender (Khalifa, 2017; Ryle, 1949/2000). La comprensión debe poder ser explicitada — juego de dar y pedir razones (Brandom, 1994/2005)—, debe ser sometida al escrutinio de los agentes que componen la subcomunidad en cuestión tanto para poder llevar a cabo la reproducción y justificación del contenido epistémico, para poder trabajar en los problemas tomados como interesantes como para poder abrir nuevos senderos de investigación. La misma condición de perfectibilidad — región heurística — de la comprensión es dependiente del carácter social y, por ende, explícito de la misma: avanzamos sobre los logros cognitivos establecidos previamente por otros usuarios.

En segundo lugar, que la comprensión debe ser evaluada en relación con los propósitos cognitivos y no en relación con su precisión o ajuste a la realidad, tal y como han tratado de defender los partidarios del veritismo (de Regt, 2017; Elgin, 2017; Giere, 2006; Parker, 2020a; Potochnik, 2017; Van Bowel, 2013; Winsberg, 2018). La comprensión no se produce cuando se revela algún nexo nómico del mundo, sino cuando se dispone de ciertas capacidades/habilidades, de cierto saber-cómo - maestría en el rol inferencial—, pudiendo satisfacer determinadas necesidades cognitivas, de manera eficaz y coherente, relativas a una determinada subcomunidad de agentes cognitivos. De esta manera, diferentes modelos, desarrollados sobre la base de diferentes objetivos cognitivos, pueden producir diferentes tipos de comprensión. Esto explica la enorme heterogeneidad de modelos científicos que hacen asunciones contradictorias. Con ello no se pretende suscribir ningún tipo de relativismo o constructivismo. La cláusula (5) está orientada a evitar este tipo de acusaciones. Para afirmar que un determinado agente A comprende un fenómeno $\mathrm{F}$, es preciso que cumpla con las siguientes condiciones de coherencia — por grado de constricción: lógica, empírica, epistémica y metodológica. La coherencia lógica establece que la comprensión debe ser lógicamente consistente. La coherencia empírica establece que debe someterse al tribunal de la experiencia. La coherencia epistémica que debe ser consistente con el cuerpo aceptado de conocimientos. La coherencia metodológica establece que debe ser consistente con las prescripciones metodológicas marcadas por la disciplina. 
En tercer lugar, que la comprensión no depende de la captación de una explicación. Como se ha podido apreciar, una suposición tradicional es que la explicación constituye el único objetivo cognitivamente relevante. Esta posición ha sido asumida tanto desde el lado veritista como desde el pragmático/contextualista. Tal y como se ha tratado de mostrar a lo largo del artículo, esta visión explicacionista de la comprensión es inadecuada, tanto desde un punto de vista descriptivo - es incapaz de describir la riqueza de los intereses cognitivos de los agentes epistémicos - como conceptual —es incapaz de delinear una noción satisfactoria de comprensión. Sostengo que la práctica científica se halla permeada por una amplia heterogeneidad de objetivos cognitivos cualitativamente distintos e igualmente valiosos (Potochnik, 2017). En la sección 2.1. se pudo observar cómo el principal objetivo cognitivo de los modelos climáticos era la proyección, la exploración de un espacio de posibilidad que permitiese a los científicos diseñar estrategias políticas, científicas y económicas de intervención sobre la realidad. Asimismo, se ha visto cómo los usuarios de los organismos modelo tenían como principal objetivo cognitivo la manipulación material de los mismos con el único fin de explorar un espacio de posibilidad que les permitiese extraer determinadas conclusiones para poder guiar investigaciones paralelas en organismos alternativos. Otra clase de objetivos cognitivos distintos de la explicación podrían ser los de clasificación — tómese el caso de la taxonomía en biología—, los pedagógicos o incluso los de unificación/síntesis de un amplio cuerpo de conocimientos a través de principios generales - tómese el caso de la Síntesis Moderna. Con esto no se pretende afirmar que la explicación no sea un objetivo cognitivo sumamente valioso $y$ deseable. Con estas afirmaciones solo se pretende resaltar dos ideas.

La primera, que existe una pluralidad de objetivos cognitivos que es preciso tener en cuenta cuando se aborda la caracterización de la comprensión científica. Con ello se pretende suscribir el principio axiológico de la tolerancia epistémica: existen objetivos cognitivos diferentes de la explicación que impulsan y motivan la comprensión. Es posible que tipos cualitativamente distintos de comprensión puedan surgir en relación con distintas necesidades cognitivas. La legitimidad de este principio axiológico viene determinada por la cláusula de efectividad $(5)^{11}$. Esto significa que lo que puede ser considerado como efectivo dentro de un contexto epistémico puede que no sea considerado en otro. Es preciso conceder a de Regt y a Victor Gijsbers el crédito de haber reconocido la importancia de implementar tal criterio $(2016,2)$. Empero, lo que no han señalado estos autores son las implicaciones que tiene dicho criterio sobre la cuestión de los grados de comprensión. La efectividad reincide en la necesidad de considerar la gradualidad horizontal de la comprensión, una gradualidad que no se articule en términos de comprensión mínima o de aproximación a una comprensión máxima (Kelp, 2017; Khalifa, 2017), sino en términos pragmáticos y contextuales, esto es, en

${ }^{11}$ Entiéndase por efectividad producir cierta clase de resultados en relación con el objetivo considerado de manera que se cumpla con las demandas cognitivas impuestas por la subcomunidad, en cierto grado. 
relación con los objetivos cognitivos de agentes particulares insertos en subcomunidades particulares. Diseñar una escala con la que comparar y clasificar, en términos absolutos, la comprensión relativa a distintos objetivos cognitivos de distintos agentes insertos en distintas subcomunidades de investigación constituye una tarea poco afortunada. Es preciso evaluar la efectividad de la comprensión en relación con unos estándares de aceptación particulares y dependientes de la subcomunidad epistémica correspondiente. Estos estándares son altamente contextuales y empíricos por lo que dar una definición precisa de los mismos resulta imposible. Lo fundamental es que la cláusula de efectividad constriñe la comprensión y que esta se debe evaluar con respecto a (Fig. 1.):

1. Las demandas cognitivas $\mathrm{D}$

2. Marcadas por una subcomunidad epistémica SC

3. En relación con un objetivo $\mathrm{O}$

4. Sobre la base del conocimiento teórico Ct y performativo Cp

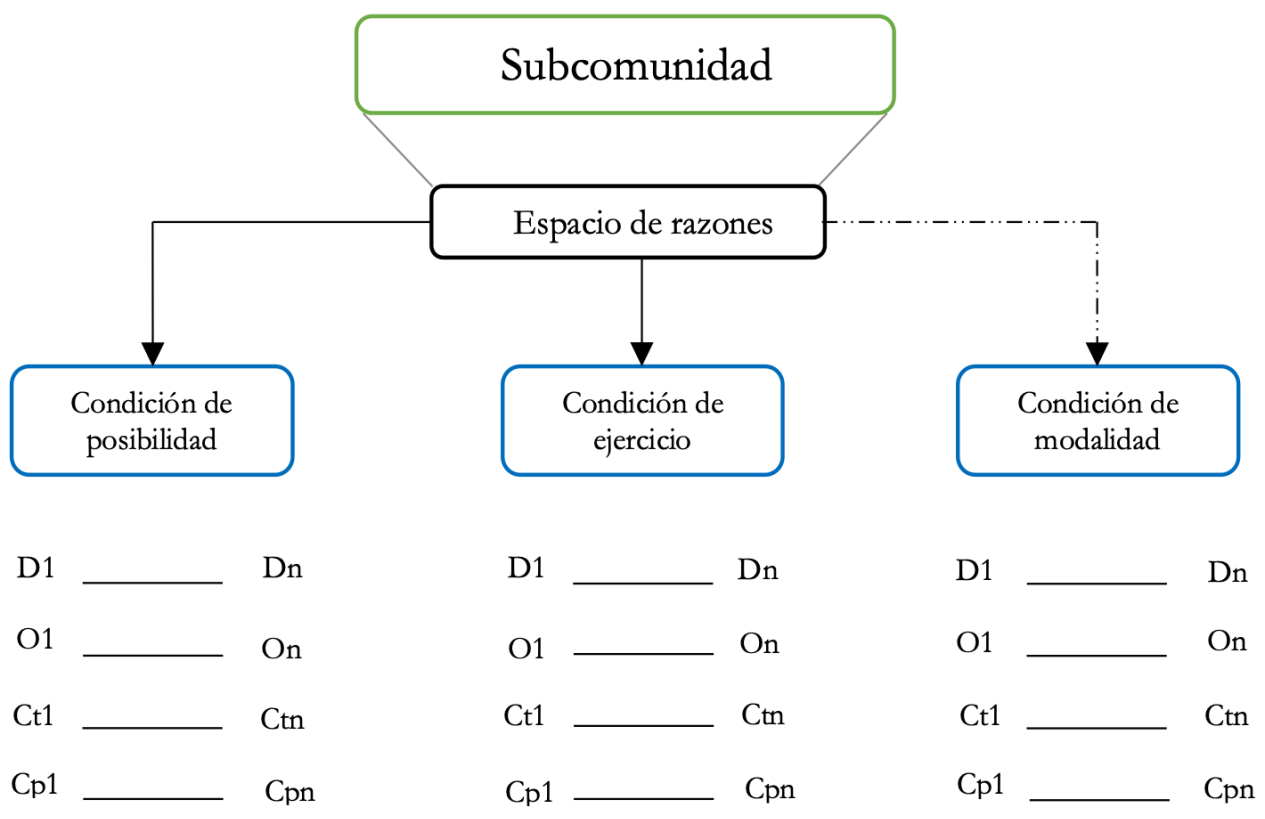

Fig. 1.

La segunda, que la comprensión no se subsume a la explicación: estos dos conceptos son independientes el uno del otro. Es decir, es posible que alguien tenga comprensión sin disponer de una explicación y que alguien disponga de una explicación sin tener comprensión. La posibilidad de comprensión sin explicación ya ha sido examinada a lo largo del artículo. En cuanto a la posibilidad de explicación sin comprensión, considere el escenario en donde un sujeto memoriza una explicación - por ejemplo, el funcionamiento del operón Lac- $\mathrm{O}$ la capta de otra persona de forma pasiva. En ambos casos, difícilmente se 
podría atribuir comprensión al agente en cuestión. Para poder afirmar que alguien ha logrado comprensión acerca de algo es preciso que el agente cognitivo en cuestión capte cuerpos de información integral (Elgin, 2017) —constelaciones de significado - , pudiendo situar — saber-cómo — dicha explicación dentro del espacio de razones de la subcomunidad correspondiente (Brandom, 2011; Rouse, 2015; Sellars, 1956/1997).

\section{Conclusión}

A lo largo del artículo se ha defendido la necesidad y la pertinencia de adoptar una actitud pragmática y contextualista con respecto a la comprensión. Para ello, se ha articulado la argumentación sobre dos estrategias fundamentales. En primer lugar, se han mostrado las principales inadecuaciones conceptuales y descriptivas de las que es presa la perspectiva veritista. Se ha establecido cómo esta postura filosófica es incapaz de abordar el marcado carácter perspectivista e idealizado de los modelos científicos. En segundo lugar, se ha evidenciado en qué medida la propuesta contextualista y pragmática de de Regt es capaz de acomodar estos dos últimos puntos. Sin embargo, se ha establecido que su teoría no era del todo satisfactoria dado que afrontaba tres problemas fundamentales. En primer lugar, era incapaz de acomodar los modelos cuyo funcionamiento era parcialmente independiente de la teoría. En segundo lugar, al ligar el concepto de comprensión al de explicación, la teoría de de Regt era incapaz de dar cuenta de la amplia variedad de objetivos cognitivos que pueblan la práctica científica. En tercer lugar, no era capaz de ofrecer una teoría de los grados de comprensión. Con el objetivo de acomodar estas críticas y poder ofrecer una propuesta pragmática viable, se ha articulado una noción deflacionaria y minimalista de comprensión basada en cinco cláusulas.

\section{Agradecimientos}

La elaboración de este artículo ha sido posible gracias al contrato predoctoral cofinanciado por la Consejería de Educación de la Junta de Castilla y León y por el Fondo Social Europeo. Agradezco los comentarios de Ana Cuevas Badallo, Juan Vicente Mayoral y de los revisores de la Revista Artefactos.

\section{Referencias bibliográficas}

Ankeny, Rachel y Leonelli, Sabina (2020). Model Organisms. Cambridge: Cambridge University Press.

Baumberger, Christoph (2019). Explicating Objectual Understanding: Taking Degrees Seriously. Journal for General Philosophy of Science, 50, 367-388.

Bertolaso, Marta (2016). Philosophy of Cancer. A Dynamic and Relational View. Springer. 
Brandom, Robert (1994/2005). Hacerlo explícito. Razonamiento, representación y compromiso discursivo. Barcelona: Herder.

Brandom, Robert (2009). Reason in Philosophy: Animating Ideas. Cambridge: Harvard University Press.

Brandom, Robert (2011). Perspectives on Pragmatism. Classical, Recent \& Contemporary. Cambridge: Harvard University Press.

Craver, Carl (2007). Explaining the Brain: Mechanisms and the Mosaic Unity of Neurosciences. Oxford: Oxford University Press.

De Regt, Henk (2009). Understanding and Scientific Explanation. En Henk de Regt, Sabina Leonelli y Kai Eigner (Eds.), Scientific Understanding. Philosophical Perspectives (pp. 21-43). Pittsburgh: University of Pittsburgh Press.

De Regt, Henk (2017). Understanding Scientific Understanding. New York: Oxford University Press.

De Regt, Henk y Dieks, Dennis (2005). A Contextual Approach to Scientific Understanding. Synthese, 144, 137-170.

De Regt, Henk y Gijsbers, Victor (2016). How False Theories can Yield Genuine Understanding. En Stephen Grimm, Christoph Baumberger y Sabine Ammon (Eds.), Explaining Understanding. New Perspectives From Epistemology and Philosophy of Science (pp. 50-75). New York: Routledge.

De Regt, Henk y Baumberger Christoph (2019). What is scientific understanding and how can it be achieved? En Kevin McKain y Kostas Kampourakis (Eds.), What is Scientific Knowledge? An Introduction to Contemporary Epistemology of Science (pp. 66-81). New York: Routledge.

Durán, Juan y Formanek, Nico (2018). Grounds for Trust: Essential Epistemic Opacity and Computational Reliabilism. Minds and Machines, 28(4), 645-666.

Elgin, Catherine (2017). True Enough. Cambridge: The MIT Press.

Elgin, Mehmet y Sober, Elliott (2002). Cartwright on explanation and idealization. Erkenntnis, 57, 441-450

Frigg, Roman, Thompson, Erica y Wendl, Charlotte (2015a). Philosophy of Climate Science Part I: Observing Climate Change. Philosophy Compass, 10(12), 953-964.

Frigg, Roman, Thompson, Erica y Wendl, Charlotte (2015b). Philosophy of Climate Science Part II: Modelling Climate Change. Philosophy Compass, 10(12), 965977.

Frigg, Roman y Nguyen, James (2019). Mirrors without warnings. Synthese.

Giere, Ronald (2006). Scientific Perspectivism. Chicago: The University of Chicago Press.

Greco, John (2014). Episteme: Knowledge and Understanding. En Kevin Timpe y Craig Boyd (Eds.), Virtues and Their Vices (pp. 285-303). Oxford: Oxford University Press. 
Grimm, Stephen (2006). Is Understanding a Species of Knowledge? Brit. J. Phil. Sci., 57(3), 515-535.

Grimm, Stephen (2008). Explanatory inquiry and the need for explanation. Br. J. Philos. Sci., 59(3), 481-497.

Grimm, Stephen (2012). The value of understanding. Philosophy Compass, 7(2), 103117.

Hacking, Ian (1983). Representing and Intervening. Introductory Topics in the Philosophy of Natural Science. Cambridge: Cambridge University Press.

Humphreys, Paul (2004). Extending Ourselves. Computational Science, Empiricism, and Scientific Method. New York: Oxford University Press.

Jones, Martin y Cartwright, Nancy (Eds.) (2005). Idealization XII: Correcting the Model. Idealization and Abstraction in the Sciences. Amsterdam: Rodopi.

Kelp, Christoph (2015). Understanding Phenomena. Synthese, 192, 3799-3816.

Kelp, Christoph (2017). Towards a knowledge-based account of understanding. En Stephen Grimm, Christoph Baumberger y Sabine Ammon (Eds.), Explaining Understanding: New Perspectives from Epistemology and Philosophy of Science (pp. 251-271). New York: Routledge.

Kelp, Christoph (2021). Inquiry, Knowledge and Understanding. Synthese, 198, 15831593. https://doi.org/10.1007/s11229-018-1803-y

Khalifa, Kareem (2017). Understanding, Explanation, and Scientific Knowledge. Cambridge: Cambridge University Press.

Kuorikoski, Jaakko y Ylikoski, Petri (2015). External representations and scientific understanding. Synthese, 192, 3817-3837.

Kvanvig Jonathan (2003). The Value of Knowledge and the Pursuit of Understanding. New York: Cambridge University Press.

Kvanvig Jonathan (2009). Responses to Critics. En Adrian Hadock, Alan Millar y Duncan Pritchard (Eds.), Epistemic Value (pp. 339-353). Oxford: Oxford University Press.

Lange, Marc (2017). Because Without Cause. Non-Causal Explanations in Science and Mathematics. Oxford: Oxford University Press.

Lenhard, Johannes (2013). The Great Deluge. Simulation Modeling and Scientific Understanding. En Henk de Regt, Sabina Leonelli y Kai Eigner (Eds.), Scientific Understanding. Philosophical Perspectives (pp. 169-189). Pittsburgh: University of Pittsburgh Press.

Lenhard, Johannes (2019). Calculated Surprises. A Philosophy of Computer Simulation. New York: Oxford University Press. 
Lenhard, Johannes y Winsberg, Eric (2010). Holism, Entrenchment, and the Future of Climate Model Pluralism. Studies in History and Philosophy of Science Part B, 41(3), 253-262.

Leonelli, Sabina (2013). Understanding in biology: The impure nature of biological knowledge. En Henk de Regt, Sabina Leonelli y Kai Eigner (Eds.), Scientific Understanding. Philosophical Perspectives (pp. 189-210). Pittsburgh: University of Pittsburgh Press.

Lipton, Peter (2013). Understanding without Explanation. En Henk de Regt, Sabina Leonelli y Kai Eigner (Eds.), Scientific Understanding. Philosophical Perspectives (pp. 169-189). Pittsburgh: University of Pittsburgh Press.

Longino, Helen (2002). The Fate of Knowledge. Princeton: Princeton University Press.

Massimi, Michaela y McCoy, Casey (Eds.) (2020). Understanding Perspectivism. Scientific Challenges and Methodological Prospects. New York: Routledge.

Morgan, Mary y Morrison, Margaret (Eds.) (1999). Models as Mediators. Perspectives on Natural and Social Science. Cambridge: Cambridge University Press.

Morrison, Margaret (2011). One Phenomenon, Many Models: Inconsistency and Complementarity. Studies in History and Philosophy of Science Part A, 42(2), 342-351.

Morrison, Margaret (2015). Reconstructing Reality: Models, Mathematics, and Simulations. Oxford: Oxford University Press.

Parker, Wendy (2006). Understanding Pluralism in Climate Modeling. Foundations of Science, 11, 349-368.

Parker, Wendy (2014). Values and uncertainties in climate prediction, revisited. Studies in History and Philosophy of Science Part A, 46, 24-30.

Parker, Wendy (2020a). Model Evaluation: An Adequacy-for-Purpose View. Philosophy of Science, 87, 457-477.

Potochnik, Angela (2017). Idealization and the Aims of Science. Chicago: The University of Chicago Press.

Price, Huw (2011). Naturalism without mirrors. Oxford: Oxford University Press.

Pritchard, Duncan (2009). 'Knowledge, Understanding and Epistemic Value'. En A. O'Hear (Ed.), Epistemology (Royal Institute of Philosophy Lectures) (pp. 19-43). Cambridge: Cambridge University Press.

Pritchard, Duncan (2010). Knowledge and Understanding. En Alan Millar y Adrian Haddock (Eds.), The Nature and Value of Knowledge: Three Investigations (pp. 1-88). Oxford: Oxford University Press.

Reutlinger, Alexander y Saatsi, Juha (Eds.) (2018). Explanation Beyond Causation: Philosophical Perspectives on Non-Causal Explanations. Oxford: Oxford University Press. 
Rice, Collin (2015). Factive Scientific Understanding without Accurate Representation. Biology \& Philosophy, 31, 81-102.

Rice, Collin (2017). Idealized models, holistic distortions, and universality. Synthese, 195, 2795-2819.

Rice, Collin (2021). Leveraging Distortions. Explanation, Idealization and Universality in Science. Cambridge: The MIT Press.

Rouse, Joseph (2015). Articulating the World: Conceptual Understanding and the Scientific Image. Chicago: The University of Chicago Press.

Sellars, Wilfrid (1956/1997). Empiricism and the Philosophy of Mind. Minneapolis: University of Minnesota Press.

Simon, Herbert (1996). The Sciences of the Artificial. Cambridge: The MIT Press.

Sosa, Ernest (2007). A Virtue Epistemology. Apt Belief and Reflective Knowledge. Oxford: Oxford University Press.

Strevens, Michael (2008). Depth: An Account of Scientific Explanation. Cambridge: Harvard University Press.

Strevens, Michael (2013). No understanding without explanation. Stud. Hist. Philos. Sci., 44, 510-515.

Suárez, Mauricio (2010). Scientific Representation. Philosophy Compass, 5(1), 91-101.

Suárez, Mauricio (Ed.) (2015). Fictions in Science. Philosophical Essays on Modeling and Idealization. New York: Routledge.

Trout, J. D. (2007). The Psychology of Scientific Explanation. Philosophy Compass, 2(3), 564-591.

Van Bowel, Jeroen (2013). Understanding in Political Science: The Plurality of Epistemic Interests. En Henk de Regt, Sabina Leonelli y Kai Eigner (Eds.), Scientific Understanding. Philosophical Perspectives (pp. 298-314). Pittsburgh: University of Pittsburgh Press.

Van Fraassen, Bas (1980). The Scientific Image. Oxford: Clarendon Press.

Weisberg, Michael (2013). Simulation and Similarity: Using Models to Understand the World. Oxford: Oxford University Press.

Winsberg, Eric (2006). Models of success versus the success of models: reliability without truth. Synthese, 152, 1-19.

Winsberg, Eric (2018). Philosophy and Climate Science. Cambridge: Cambridge University Press.

Winsberg, Eric (2019). Computer Simulations in Science. The Stanford Encyclopedia of Philosophy (Winter 2019 Edition), Edward N. Zalta (Ed.). https://plato.stanford. edu/entries/simulations-science/ 
Mariano Martín Villuendas

¿Es necesaria la verdad? Una noción pragmática y deflacionaria de comprensión

Winther, Rasmus (2020). When Maps become the World. Chicago: The University of Chicago Press.

Wittgenstein, Ludwig (2015). Los cuadernos azul y marrón. Madrid: Tecnos.

Woodward, James (2003). Making Things Happen. A Theory of Causal Explanation. New York: Oxford University Press. 